Research Paper

\title{
The Spectrum of Malignant Neoplasms among Liver Transplant Recipients: Sociodemographic Factors, Mortality, and Hospital Burden
}

\author{
Maryam Haider, MD ${ }^{\bowtie}$; Anusha Bapatla, MD; Rana Ismail, PhD, MSc; Ahmed J Chaudhary, MD, FACP; \\ Sana Iqbal, MD, FACP; Syed M Haider, MS \\ Department of Internal Medicine, Detroit Medical Center/Wayne State University-Sinai Grace Hospital, Detroit, USA. \\ $\triangle$ Corresponding author: Maryam Haider, MD, Department of Internal Medicine, Detroit Medical Center/Wayne State University Sinai Grace Hospital. 6071 \\ Outer Dr W, Detroit, MI 48235. Tel: +15856267058. E-mail: maryambilalhaider@yahoo.com \\ (c) The author(s). This is an open access article distributed under the terms of the Creative Commons Attribution License (https://creativecommons.org/licenses/by/4.0/). \\ See http://ivyspring.com/terms for full terms and conditions.
}

Received: 2021.08.26; Accepted: 2021.12.27; Published: 2022.01.09

\begin{abstract}
Objective: To determine the nationwide prevalence of malignant neoplasms (excluding hepatocellular carcinoma-HCC) in hospitalized liver transplant recipients and to study the hospital utilization, and mortality to the incidence of malignancies. To the best of our knowledge, few epidemiological studies addressed outcomes in post-liver transplant patients, such as the annual number of hospitalizations, mortality, patient characteristics regarding malignancies.

Methods: NIS database was queried between 2016 and 2018 to retrieve records of patients admitted with a principal or secondary diagnosis of liver transplant following the International Classification of Diseases, tenth Revision (ICD-I0). The population was divided into case and control groups according to the presence and absence of malignant neoplasm (MN) except for HCC. We also compared the incidence of MN in LTX patients and non-LTX matched cohort.

Results: A total of $7.28 \%$ admissions were associated with malignant neoplasms (except HCC) in LTX patients. Lymphomas, respiratory, gastrointestinal (excluding HCC), leukemia, and head/neck were commonest cancers with estimated admission rates of $0.97 \%, 0.90 \%, 0.80 \%, 0.53 \%$, and $0.49 \%$, respectively. Lung cancer was the most frequent malignant neoplasm among White and Black racial/ethnic groups (15.78\% and 14.8\%), whereas lymphoma was pervasive among Hispanics (20.3\%). Lung cancer had the highest in-hospital mortality (10.55\%), followed by the cancer of the nervous system (9.09\%). The LTX and non-LTX cohort comparison showed that LTX patients are at increased risk of head and neck cancers, skin cancers, lymphomas, tumors, and Myelodysplastic syndrome. According to a multivariate analysis, a statistically significant association existed between malignant neoplasms in LTX patients and the following factors: increasing age $(P<.001)$, higher mortality $(P<.001)$, females with $29 \%$ lesser odds than males $(P<.001)$, Black race and Hispanic ethnicity with $20 \%$ and $26 \%$ lesser odds as compared to White $(P<.05)$. Clinical factors included smoking, Alcoholic cirrhosis, Hepatitis $B$, and Hepatitis $C$, were statistically significant risk factors of post-liver transplantation malignancies.

Conclusions: Malignancies were frequent among elderly patients and predominantly in males. Lymphoproliferative diseases were the most prevalent malignancy types, followed by respiratory/lung cancerwhich showed the highest mortality risk of all cancers. LTX patients are at increased risk of head and neck cancers, skin cancers, lymphoma, tumors, and Myelodysplastic syndrome compared to non-LTX patients.
\end{abstract}

Key words: Liver Transplantation, Transplant Recipients, Neoplasms, hepatocellular carcinoma (HCC), Lymphoma, Gastrointestinal Neoplasms.

\section{Introduction}

Liver transplantation (LTX) is the second most common organ transplant after Kidney transplantation; 2017 alone witnessed 8,000 liver transplants, and since 2016, 80,000 adults have been living with functional liver grafts [1]. Post-liver transplant complications include acute or chronic graft rejection, adverse events associated with immunosuppressive medications, and primary liver disease recurrence. 
Since long-term immunosuppressive agents are required post-transplantation, their main adverse effect consists of developing malignancies. The literature provides ample evidence that prolonged immunosuppression is associated with carcinogenesis [2-3]. Some studies showed that the most frequent de novo malignancies (DNMs) in adult LTX recipients are skin, followed by lymphoproliferative diseases [4]. Other malignancies include lung cancer, colorectal carcinoma, head and neck cancers [5]. Skin cancers are common post-transplant; they are usually localized and respond to local treatment [6], and do not require hospital admission. The risk of developing colorectal cancer is 2 to 3 times higher in LTX recipients, and it increases exponentially in patients getting transplantation secondary to primary biliary sclerosing cholangitis [7].

To the best of our knowledge, few epidemiological studies addressed outcomes in post-liver transplant patients, such as the annual number of hospitalizations, mortality, patient characteristics, and malignancies. This study aimed to determine the nationwide prevalence of malignant neoplasms, and the associated trends, clinical risk factors, patient demographics in hospitalized LTX patients. In addition, we aimed to compare the incidence of malignancies in LTX and non-LTX patients.

\section{Materials and Methods}

\section{Data Source}

We used the National Inpatient Sample (NIS) database of hospitalized patients in the United States with data collected between January 2016 and December 2018, and we only selected data on patients with a primary or secondary diagnosis of liver transplant (LTX) based on an ICD-10 (International Classification of Diseases, tenth Revision) code of Z944. All malignant neoplasms were also identified with ICD-10 codes and categorized into Head and Neck, Gastrointestinal (excluding Liver), Bone, Skin, Breast, Reproductive system, Urinary system, Nervous system, Endocrine system, Lymphoma, Leukemia, Myeloma, Tumors, Myelodysplastic Syndrome, Cancer of other sites and Secondary malignancies. The NIS database houses more than 8 million records per year. It is the largest all-payer dataset from the Healthcare Cost and Utilization Project (HCUP), maintained by the Agency of Healthcare Research Quality (AHRQ) [11]. NIS upgraded the diagnosis coding from ICD-9 to ICD-10 in September 2015 and redesigned their sampling techniques and weights on participating hospitals' data to optimize accuracy by reducing the margin of error in statistical estimates of outcomes, thus, generating more representation of national estimates. In the database, each patient can have up to 40 diagnoses depending on the dataset year. The dataset is publicly available and lacks patient identification information; thus, Institutional Review Board-IRB approval or informed consent was unnecessary under the Health Insurance Portability and Accountability Act-HIPAA [12].

\section{Variables and Outcomes}

The primary outcome included the prevalence of malignant neoplasms and the corresponding correlation with sociodemographic risk factors in LTX patients. The main variables consisted of patients and hospital characteristics, including age, sex, race, clinical risk factors, socioeconomic status, admission type, admission day, hospital bed size, hospital location, and region. The study population was divided into cases (LTX with malignant neoplasms-except for HCC) and controls (LTX without malignant neoplasms). HCC is considered a common indication for LTX, so we excluded it from the data analysis to avoid bias. The secondary outcomes analyzed the hospital utilization, including discharge status, inpatient mortality, length of stay (LOS), and inpatient hospital-related total cost of care for all malignant neoplasms. The secondary outcome also includes a comparative analysis of the incidence of malignancies among LTX and non-LTX patients.

\section{Statistical Analysis}

The statistical analyses were performed using the SAS statistical software (SAS Institute Inc., Cary, NC, United States). We used mean ( \pm standard deviation-SD) or median (interquartile range-IQR= Q3-Q1) for continuous variables such as age, total charges, and length of stay (LOS). Percentages denoted categorical variables. We performed group comparisons based on sociodemographic characteristics (age, sex, race, median socioeconomic status by national quartile) using Student t-test (for continuous variables) and Chi-square tests (for categorical variables). We used t-tests to calculate the difference in LOS and total charges. Age was categorized into five groups for group-level comparisons $(<18$; 18-49; $50-59 ; 60$ to 69 ; and $>=70$ years of age). A multivariate model developed from the stepwise logistic regression was used to test the predictor variables' association with malignant neoplasms. This regression model selected the most relevant variables to retain at a significant effect level of entry of 0.15 and a level of staying of 0.10 , removing other variables not fitting this effect criterion. We calculated odds ratios from logistic regression models, examined covariates' effects on the predictor variables, and adjusted these 
odds ratios (adjusted aOR) for confounders such as age, gender, and race. We selected a non-LTX matched group based on age, sex, and race using the 1:1 ratio nearest neighbor (greedy) propensity score method. The goodness of fit model was evaluated with Pearson's Chi-square. All hypothesis testing used a two-tailed p-value with the significance level set at 0.05 . The missing data on race, primary payer, median household income, discharge status, hospital location, and hospital teaching status were labeled with "other" or "Unknown".

\section{Results}

A total of 26225 hospital admissions occurred between 2016 and 2018 with liver transplant status, of which 1909 (7.28\%) entries were associated with malignant neoplasms (except HCC)- thus constituted our group of cases of hospitalized LTX patients.

\section{Admission Rate of Malignant Neoplasms}

Lymphomas were the most prevalent cancer among LTX patients, followed by respiratory cancers, gastrointestinal cancers (excluding liver or HCC), leukemia, and head/neck cancers with estimated admission rates of $0.97 \%, 0.90 \%, 0.80 \%, 0.53 \%$, and $0.49 \%$, respectively. Cardiac cancers were the least prevalent, followed by cancers of the nervous system and bone (Figure 1).

\section{Patient Demographics and clinical risk factors}

Malignant neoplasms with LTX were more frequent in older patients with a mean age of 61.21 years (SD \pm 15.08 ) and among males (68.73\% among cases vs. $59.20 \%$ among controls) than females (31.27\% for cases vs. $40.80 \%$ for controls) (Table $\mathbf{1}$ ). Respiratory cancer was the most prevalent malignant neoplasm among White patients $(15.78 \%)$, followed by lymphoma $(14.33 \%)$ and gastrointestinal cancer $(12.29 \%)$. Among Black patients, respiratory cancer $(14.81 \%)$, reproductive system cancer $(12.96 \%)$, and leukemia $(11.11 \%)$ were the most reported. Among Hispanics, lymphoma $(20.27 \%)$ and gastrointestinal cancers (13.51\%) prevailed (Figure 2).

Malignant neoplasms with LTX were more frequent in patients with smoking (37.56\% among cases vs. $32.10 \%$ among controls), $p<.01$, alcoholic cirrhosis $(3.51 \%$ among cases vs. $2.61 \%$ among controls),$p<.05$, hepatitis B $(3.09 \%$ among cases vs. $1.69 \%$ among controls $), p<.01$, hepatitis C $(12.94 \%$ among cases vs. $9.83 \%$ among controls),$p<.01$, and opioids $(3.72 \%$ among cases vs. $2.94 \%$ among controls), $p=0.05$. Immunosuppressants, HIV and Primary sclerosing cholangitis (PSC) were also more frequent in patients with LTX but was found to be statistically not significant.
Table 1: Baseline Patient Demographics with Liver Transplant, with and without Malignant Neoplasms.

\begin{tabular}{|c|c|c|c|}
\hline \multirow[t]{2}{*}{ Variables } & \multicolumn{2}{|c|}{ Malignant Neoplasms } & \multirow{2}{*}{$\begin{array}{l}P \\
- \text { value }\end{array}$} \\
\hline & $\begin{array}{l}\text { No } \\
(\mathrm{n}=24316) 92.72 \%\end{array}$ & $\begin{array}{l}\text { Yes } \\
(\mathrm{n}=1909) 7.28 \%\end{array}$ & \\
\hline Sex & & & $<0.01^{2}$ \\
\hline Female & $9922(40.80 \%)$ & $597(31.27 \%)$ & \\
\hline Male & $14394(59.20 \%)$ & $1312(68.73 \%)$ & \\
\hline Age (y), mean (SD) & $55.23(18.99)$ & $61.21(15.08)$ & $<0.01^{1}$ \\
\hline \multicolumn{4}{|l|}{ Age groups (y) } \\
\hline$<=17$ & $1829(7.52 \%)$ & $72(3.77 \%)$ & \\
\hline $18-49$ & $4236(17.42 \%)$ & $165(8.64 \%)$ & \\
\hline $50-59$ & $4984(20.50 \%)$ & $359(18.81 \%)$ & \\
\hline $60-69$ & $8923(36.70 \%)$ & $814(42.64 \%)$ & \\
\hline$>=70$ & $4344(17.86 \%)$ & $499(26.14 \%)$ & \\
\hline Race/Ethnicity & & & $<0.01^{3}$ \\
\hline White & $15885(65.33 \%)$ & $1356(71.03 \%)$ & \\
\hline Black & $2281(9.38 \%)$ & $129(6.76 \%)$ & \\
\hline Hispanic & $3366(13.84 \%)$ & $192(10.06 \%)$ & \\
\hline Asian or Pacific Islander & $652(2.68 \%)$ & $67(3.51 \%)$ & \\
\hline Native American & $182(0.75 \%)$ & $9(0.47 \%)$ & \\
\hline Other & $1950(8.02 \%)$ & $156(8.17 \%)$ & \\
\hline $\begin{array}{l}\text { Median socioeconomic status by } \\
\text { national quartiles }\end{array}$ & & & $<0.01^{3}$ \\
\hline $0-25$ & $6132(25.22 \%)$ & $401(21.01 \%)$ & \\
\hline $25-50$ & $6158(25.32 \%)$ & $475(24.88 \%)$ & \\
\hline $50-75$ & $6300(25.91 \%)$ & $565(29.60 \%)$ & \\
\hline $75-100$ & $5303(21.81 \%)$ & $447(23.42 \%)$ & \\
\hline Other & $423(1.74 \%)$ & $21(1.10 \%)$ & \\
\hline Admission Type & & & $<0.01^{2}$ \\
\hline Non-elective & $20921(86.16 \%)$ & $1492(78.24 \%)$ & \\
\hline Elective & $3361(13.84 \%)$ & $417(21.76 \%)$ & \\
\hline Admission Day & & & $<0.01^{2}$ \\
\hline Weekdays & $19421(79.87 \%)$ & $1574(82.45 \%)$ & \\
\hline Weekend & $4895(20.13 \%)$ & $335(17.55 \%)$ & \\
\hline Hospital Bed Size & & & $<0.01^{3}$ \\
\hline Small & $2961(12.18 \%)$ & $185(9.69 \%)$ & \\
\hline Medium & $5525(22.72 \%)$ & $409(21.42 \%)$ & \\
\hline Large & $15830(65.10 \%)$ & $1315(68.88 \%)$ & \\
\hline $\begin{array}{l}\text { Location/teaching status of } \\
\text { hospital }\end{array}$ & & & NS \\
\hline Rural & $1030(4.24 \%)$ & $76(3.98 \%)$ & \\
\hline Urban nonteaching & $3250(13.37 \%)$ & $218(11.42 \%)$ & \\
\hline Urban teaching & $20036(84.40 \%)$ & $1615(84.60 \%)$ & \\
\hline Hospital region & & & NS \\
\hline Northeast & $4505(18.53 \%)$ & $414(21.69 \%)$ & \\
\hline Midwest or North Central & $5558(22.86 \%)$ & $441(23.10 \%)$ & \\
\hline South & $9318(38.32 \%)$ & $679(35.57 \%)$ & \\
\hline West & $4935(20.30 \%)$ & $375(19.64 \%)$ & \\
\hline Discharge Characteristics & & & $<0.01^{3}$ \\
\hline Routine Discharge & $15714(64.92 \%)$ & $1072(56.16 \%)$ & \\
\hline Transfer to Short-term Hospital & $1093(4.49 \%)$ & $76(3.98 \%)$ & \\
\hline Transfer to other facilities & $3108(12.78 \%)$ & $234(12.26 \%)$ & \\
\hline Home Health Care (HHC) & $4008(16.48 \%)$ & $423(22.16 \%)$ & \\
\hline In hospital mortality & $393(1.62 \%)$ & $104(5.45 \%)$ & \\
\hline Length of stay (days), mean (SD) & $5.5617(7.94)$ & $6.4547(8.54)$ & $<0.01^{1}$ \\
\hline Total charges (USD), mean (SD) & $64994(115960)$ & 77127 (102652) & $<0.01^{1}$ \\
\hline \multicolumn{4}{|l|}{ Malignancies Risk Factors } \\
\hline Smoking & $7806(32.10 \%)$ & $717(37.56 \%)$ & $<0.01^{2}$ \\
\hline Alcoholic cirrhosis & $634(2.61 \%)$ & $67(3.51 \%)$ & 0.0185 \\
\hline Hepatitis B & $410(1.69 \%)$ & $59(3.09 \%)$ & $<0.01^{2}$ \\
\hline Hepatitis C & $2390(9.83 \%)$ & $247(12.94 \%)$ & $<0.01^{2}$ \\
\hline HIV & $120(0.49 \%)$ & $12(0.63 \%)$ & NS \\
\hline Immunosuppressants & $183(0.75 \%)$ & $16(0.84 \%)$ & NS \\
\hline Opioid Use & $715(2.94 \%)$ & $71(3.72 \%)$ & 0.05 \\
\hline Primary sclerosing cholangitis & $56(0.23 \%)$ & $6(0.31 \%)$ & NS \\
\hline
\end{tabular}

${ }^{1}$ Two sample Student $t$-test, 2-tailed for comparing means of two Continuous Variables. ${ }^{2}$ Pearson Chi-Square 2-tailed Test for association of two Categorical

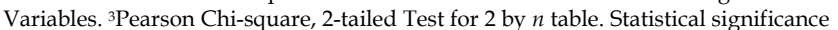
illustrates that two group differs. NS: Not statistically significant. 


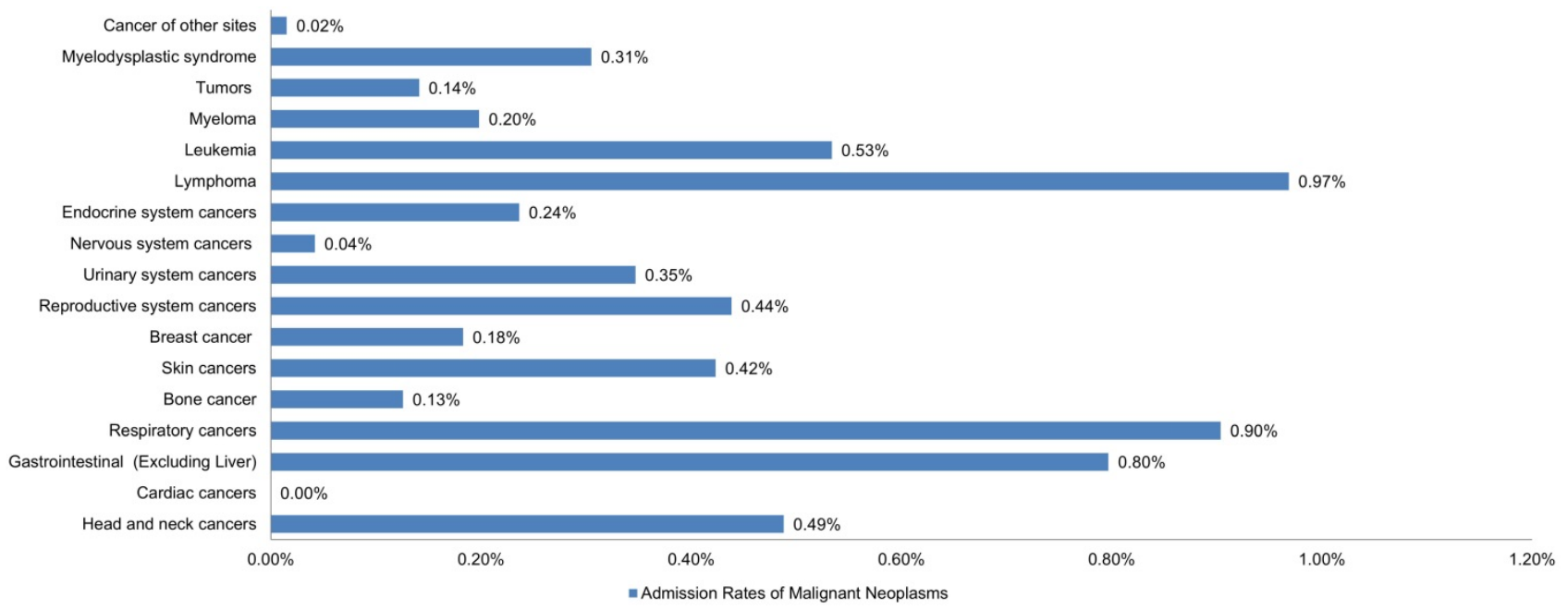

Figure 1: Bar Chart of Admission Rates of Malignant Neoplasms in Liver Transplant Patients.

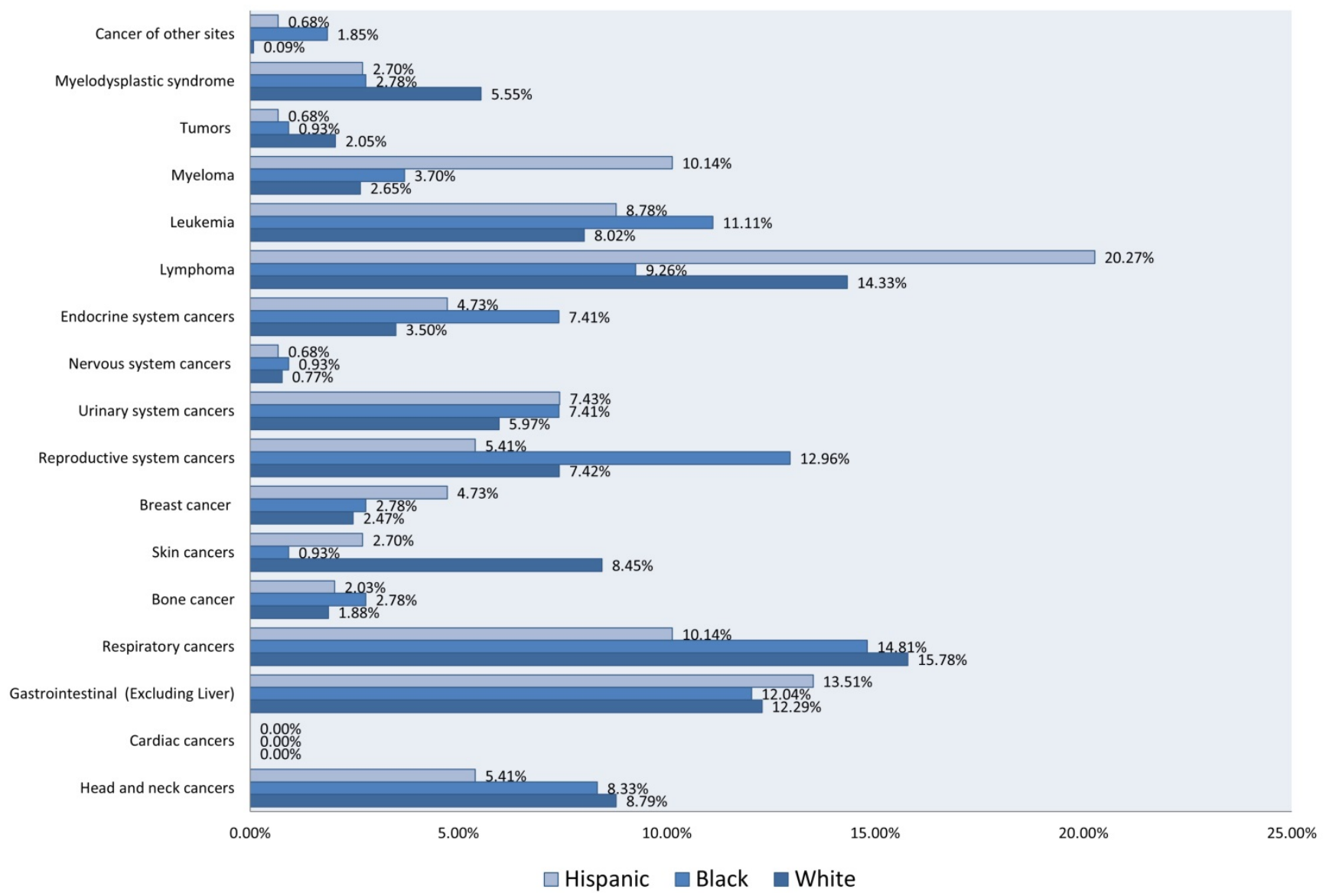

Figure 2: Bar Chart of Rates of Malignant Neoplasms in 1677 Patients, Stratified by Race and Ethnicity. (1356 White, 129 Black, and 192 Hispanic).

Table 2 showed the univariate and multivariate regression analyses and some selected sociodemographic and clinical risk factors of malignant neoplasms in hospitalized LTX patients. The multivariate logistic regression showed that female patients were $29 \%$ less likely to develop cancers compared to males (aOR, 0.712; 95\% CI, 0.643 - 0.788; $p<.001)$. Compared to patients aged 17 years or less, the odds of cancers in patients aged 18-49 were the same; however, in patients aged 50-59, the odds of cancers were 1.68 times higher $(a O R, 1.683 ; 95 \% \mathrm{CI}$, $1.294-2.190) ; p<.001), 2.1$ times higher in patients aged 60-69 (aOR, 2.091; 95\% CI, $1.625-2.690 ; p<.001)$, and 2.69 times higher in those aged over $70(a O R$, 2.685; 95\% CI, $2.067-3.488 ; p<.001$ ). In terms of race, compared to White patients, Black patients had $20 \%$ 
$(a O R, 0.802 ; 95 \% \mathrm{CI}, 0.663-0.970 ; p<.05)$ and Hispanic patients had $24 \%$ lesser odds ( $a O R, 0.759$; 95\% CI, 0.647- 0.889; $p$.001) of developing malignant neoplasms. Asian or Pacific Islander and Native American odds were not statistically significant. The median household income using national quartiles ranging from 1 (lowest median income) to 4 (highest median income) was utilized in the regression model as a proxy measure reflecting patients' socioeconomic status based on their communities' zip codes. Compared to median household income in quartile 1, the odds of cancers in patients with median household income in quartile 3 were 1.28 times $(a O R$,
1.285; 95\% CI, $1.121-1.473 ; p<.001)$, and 1.16 times $(a O R, 1.161 ; 95 \%$ CI, $1.004-1.342 ; p<.05)$ in quartile 4.

LTX Patients have $12 \%$ greater odds to develop cancers with smoking (aOR, 1.121; 95\% CI, 1.013 $1.240 ; p<.05), 30 \%$ greater odds with alcoholic cirrhosis $(a O R, 1.1298 ; 95 \% \mathrm{CI}, 1.0-1.687 ; p=.051)$, $54 \%$ greater odds with hepatitis B $(a O R, 1.554 ; 95 \%$ CI, $1.166-2.071 ; p<.01), 24 \%$ greater odds with hepatitis C (aOR, 1.237; 95\% CI, 1.069- 1.431; $p<.01)$, and 36\% greater odds with opiods $(a O R, 1.362 ; 95 \% \mathrm{CI}, 1.056$ $1.756 ; p<.01)$.

Table 2: Risk Factors of Malignant Neoplasms in Hospitalized Patients with Liver Transplant Status in the United States- from 2016 to 2018.

\begin{tabular}{|c|c|c|c|c|}
\hline Malignant Neoplasms & Odds Ratio (95\%CI) & $P$ value & Adjusted Odds Ratio $(95 \% \mathrm{CI})$ & $P$ value \\
\hline & Univariate logistic regression ${ }^{1}$ & & Multivariate logistic regression ${ }^{2}$ & \\
\hline Sex, Female vs Male & $0.660(0.597-0.730)$ & $<.001$ & $0.712(0.643-0.788)$ & $<.001$ \\
\hline \multicolumn{5}{|l|}{ Age groups (y) } \\
\hline$<=17$ & Reference & NA & Reference & NA \\
\hline $18-49$ & $0.989(0.746-1.312)$ & NS & $0.976(0.734-1.299)$ & NS \\
\hline $50-59$ & $1.830(1.413-2.370)$ & $<.001$ & $1.683(1.294-2.190)$ & $<.001$ \\
\hline $60-69$ & $2.317(1.812-2.964)$ & $<.001$ & $2.091(1.625-2.690)$ & $<.001$ \\
\hline$>=70$ & $2.918(2.265-3.758)$ & $<.001$ & $2.685(2.067-3.488)$ & $<.001$ \\
\hline Race/ethnicity & & & & $<.001$ \\
\hline White & Reference & NA & Reference & NA \\
\hline Black & $0.663(0.550-0.798)$ & $<.001$ & $0.802(0.663-0.970)$ & $<.05$ \\
\hline Hispanic & $0.668(0.572-0.781)$ & $<.001$ & $0.759(0.647-0.889)$ & $<.001$ \\
\hline Asian or pacific islander & $1.204(0.931-1.557)$ & NS & $1.219(0.938-1.585)$ & NS \\
\hline Native American & $0.937(0.789-1.113)$ & NS & $0.789(0.401-1.552)$ & NS \\
\hline \multicolumn{5}{|c|}{ Median socioeconomic status by national quartiles } \\
\hline $0-25$ & Reference & NA & Reference & NA \\
\hline $25-50$ & $1.180(1.028-1.353)$ & NS & $1.117(0.971-1.285)$ & NS \\
\hline $50-75$ & $1.371(1.201-1.566)$ & $<.001$ & $1.285(1.121-1.473)$ & $<.001$ \\
\hline $75-100$ & $1.289(1.121-1.482)$ & NS & $1.161(1.004-1.342)$ & $<.05$ \\
\hline Admission Type, Elective vs Non-elective & $1.731(1.544-1.942)$ & $<.001$ & $1.731(1.540-1.946)$ & $<.001$ \\
\hline \multicolumn{5}{|l|}{ Hospital Bed Size } \\
\hline Small & Reference & NA & Reference & NA \\
\hline Medium & $1.185(0.990-1.417)$ & NS & $1.277(1.065-1.532)$ & $<.005$ \\
\hline Large & $1.329(1.134-1.558)$ & $<.001$ & $1.473(1.253-1.732)$ & $<.001$ \\
\hline \multicolumn{5}{|l|}{ Location/teaching status of hospital } \\
\hline Rural & Reference & NA & Reference & NA \\
\hline Urban nonteaching & $0.909(0.694-1.191)$ & NS & $0.883(0.669-1.164)$ & NS \\
\hline Urban teaching & $1.092(0.861-1.387)$ & NS & $1.162(0.908-1.487)$ & NS \\
\hline \multicolumn{5}{|l|}{ Hospital region } \\
\hline Northeast & Reference & NA & Reference & NA \\
\hline Midwest or North Central & $0.863(0.751-0.993)$ & NS & NA & NS \\
\hline South & $0.793(0.698-0.901)$ & $<.001$ & NA & NS \\
\hline West & $0.827(0.715-0.956)$ & NS & NA & NS \\
\hline \multicolumn{5}{|l|}{ Discharge Characteristics } \\
\hline Routine Discharge & Reference & NA & Reference & NA \\
\hline Transfer to Short-term Hospital & $1.019(0.801-1.297)$ & NS & $1.093(0.856-1.397)$ & NS \\
\hline Transfer to other facilities & $1.104(0.953-1.278)$ & NS & $0.951(0.818-1.106)$ & NS \\
\hline Home Health Care (HHC) & $1.547(1.375-1.740)$ & $<.001$ & $1.342(1.190-1.514)$ & $<.001$ \\
\hline Smoking & $1.273(1.156-1.402)$ & $<.001$ & $1.121(1.013-1.240)$ & $<.05$ \\
\hline Alcoholic cirrhosis & $1.360(1.053-1.757)$ & 0.0186 & $1.298(1.0-1.687)$ & 0.0507 \\
\hline Hepatitis B & $1.860(1.410-2.454)$ & $<.001$ & $1.554(1.166-2.071)$ & $<.01$ \\
\hline Hepatitis C & $1.365(1.186-1.570)$ & $<.001$ & $1.237(1.069-1.431)$ & $<.01$ \\
\hline HIV & $1.276(0.704-2.314)$ & NS & NA & NS \\
\hline Immunosuppression & $1.115(0.667-1.863)$ & NS & NA & NS \\
\hline Opioid & $1.276(0.995-1.636)$ & 0.0546 & $1.362(1.056-1.756)$ & $<.05$ \\
\hline PSC & $1.367(0.589-3.177)$ & NS & NA & NS \\
\hline
\end{tabular}

1Univariate logistic regression is performed in SAS software with PROC Logistic.

${ }^{2}$ Mutivariate logistic regression is performed with stepwise logistic regression with a 0.15 significance level of entry and 0.10 significance level of stay. NS: Not statistically significant. 
Patients with malignant neoplasms had 73\% greater odds of elective admissions than non-elective admissions (aOR, 1.731; 95\% CI, 1.540 - 1.946; $p<$ .001 ), and $27 \%$ more likely to get admitted to medium hospitals as compared to small $(a O R, 1.277 ; 95 \% \mathrm{CI}$, 1.065 - 1.532; $p<.005)$, and higher likelihood to get admitted into large hospitals $(47 \%)$ than to small $(a O R, 1.473 ; 95 \%$ CI, 1.253 - 1.732; $p<.005)$. In our study, the hospital's teaching status and its region failed to show a statistically significant association with risk for malignant neoplasm. Regarding discharge characteristics, cases were $34 \%$ more likely to transfer to home health care (HHC) as compared to routine discharges $(a O R, 1.342 ; 95 \% \mathrm{CI}, 1.190$ - 1.514; $p$ $<.001$ ), and had 3.9 times greater odds of in-hospital mortality $(a O R, 3.293$; 95\% CI, $2.616-4.145 ; p<.001)$. The mean differences in total length of hospital stay (LOS) and hospital inpatient charges were found to be higher in LTX patients with malignant neoplasms $(0.89$; 95\% CI, $0.52-1.26$; $p$.001) and $(\$ 12132$ 95\% CI, $\$ 6757$ - \$17508; $p<.001)$, respectively.

\section{Incidence of Malignant Neoplasms in LTX patients and matched Cohort of Non-LTX patients}

Table 3 showed the incidence of Malignant Neoplasms in LTX patients and a matched cohort of non-LTX patients. We selected a matched cohort utilizing propensity score method based on patients age, sex, and race. The comparison showed that LTX patients are at increased risk of head and neck cancers, skin cancers, lymphoma's, tumors, and Myelodysplastic syndrome. The incidence of head and neck cancer after liver transplantation is 1.5 times higher $(0.49 \%$ among LTX vs. $0.33 \%$ non-LTX; $p<.01)$, 3.2 times higher in skin cancer $(0.49 \%$ among LTX vs. $0.33 \%$ non-LTX; $p<.01), 1.4$ times higher in lymphoma's $(0.97 \%$ among LTX vs. $0.69 \%$ non-LTX; $p$ $<.01), 1.58$ times higher in Myelodysplastic syndrome $(0.30 \%$ among LTX vs. $0.19 \%$ non-LTX; $p<.01)$, and 1.55 times higher in tumors $(0.14 \%$ among LTX vs. $0.09 \%$ non-LTX; $p=.096$ ).

\section{Hospital Utilization and Discharge Characteristics}

Most hospital admissions were non-elective (78.24\% among cases vs. $86.16 \%$ among controls) as compared to elective $(21.76 \%$ among cases and $13.84 \%$ among controls), $p<.001$, more patients admitted on weekdays- $80 \%$ compared to around $20 \%$ during weekdays and weekends, respectively in both cases and controls $(p<.001)$. In both groups, more than $65 \%$ of patients got admitted to large hospitals $(68.88 \%$ of cases vs. $65.10 \%$ of controls) compared to $21 \%$ to medium hospitals and $10 \%$ to small hospitals $(p<$ .001). In terms of hospital teaching status, most patients with LTX preferred admission to an urban teaching hospital $(84.60 \%$ for cases and $84.40 \%$ for controls) than urban nonteaching and rural hospitals (Table 1). Patients with LTX and malignant neoplasms (cases) had a higher length of stay (LOS) compared to LTX patients (controls), $M=6.45$ days $(S D \pm 8.54)$ vs. $M=5.56$ days $(S D \pm 7.94)$, respectively (Table 4). Similarly, Table 4 showed that cases had a higher inpatient hospital cost of care than controls with a respective mean total charge of $\$ 77,127$ (SD $\pm \$ 102,652)$ vs. $\$ 64994$ (SD $\pm \$ 115,960)$. Patients admitted with bone, gastrointestinal, head, neck, and myeloma cancers had a higher mean length of stay (LOS) than other cancers (Figure 3). In terms of total charges, malignant bone neoplasm had the highest total charges, followed by head and neck cancers, nervous system cancers, and myeloma (Figure 4). Respiratory neoplasm had the highest in-hospital mortality followed by cancer of the nervous system, myelodysplastic syndrome, and breast cancers with a mortality rate of $10.55 \%, 9.09 \%, 8.75 \%$, and $8.33 \%$, respectively (Figure 5). For discharge status, all cases had the highest routine discharges and lowest discharges to short-term hospitals. Furthermore, most cases witnessed a higher transfer rate to home healthcare than transfer to other facilities (including Skilled Nursing Facility and Intermediate Care Facility) - except for nervous system cancers and myeloma (Figure 6).

Table 3: Comparison of inpatient mortality, mean total charges and length of stay between liver transplant patients with malignant neoplasms and liver transplant patients without malignant neoplasms, 2016-2018.

\begin{tabular}{|c|c|c|c|c|c|c|}
\hline & Mortality & & Length of Stay & & Total Charges & \\
\hline & Adjusted Odds Ratio $(95 \% \mathrm{CI})$ & $P$ value & Difference $(95 \% \mathrm{CI})$ & $P$ value & Difference $(95 \% \mathrm{CI})$ & $P$ value \\
\hline LTX with Malignant Neoplasms & $3.293(2.616-4.145)$ & $<.001$ & $0.8930(0.5210-1.2650)$ & $<.001$ & $\$ 12132.8(\$ 6757-\$ 17508)$ & $<.001$ \\
\hline LTX without Malignant Neoplasms & Reference & NA & Reference & NA & Reference & NA \\
\hline
\end{tabular}




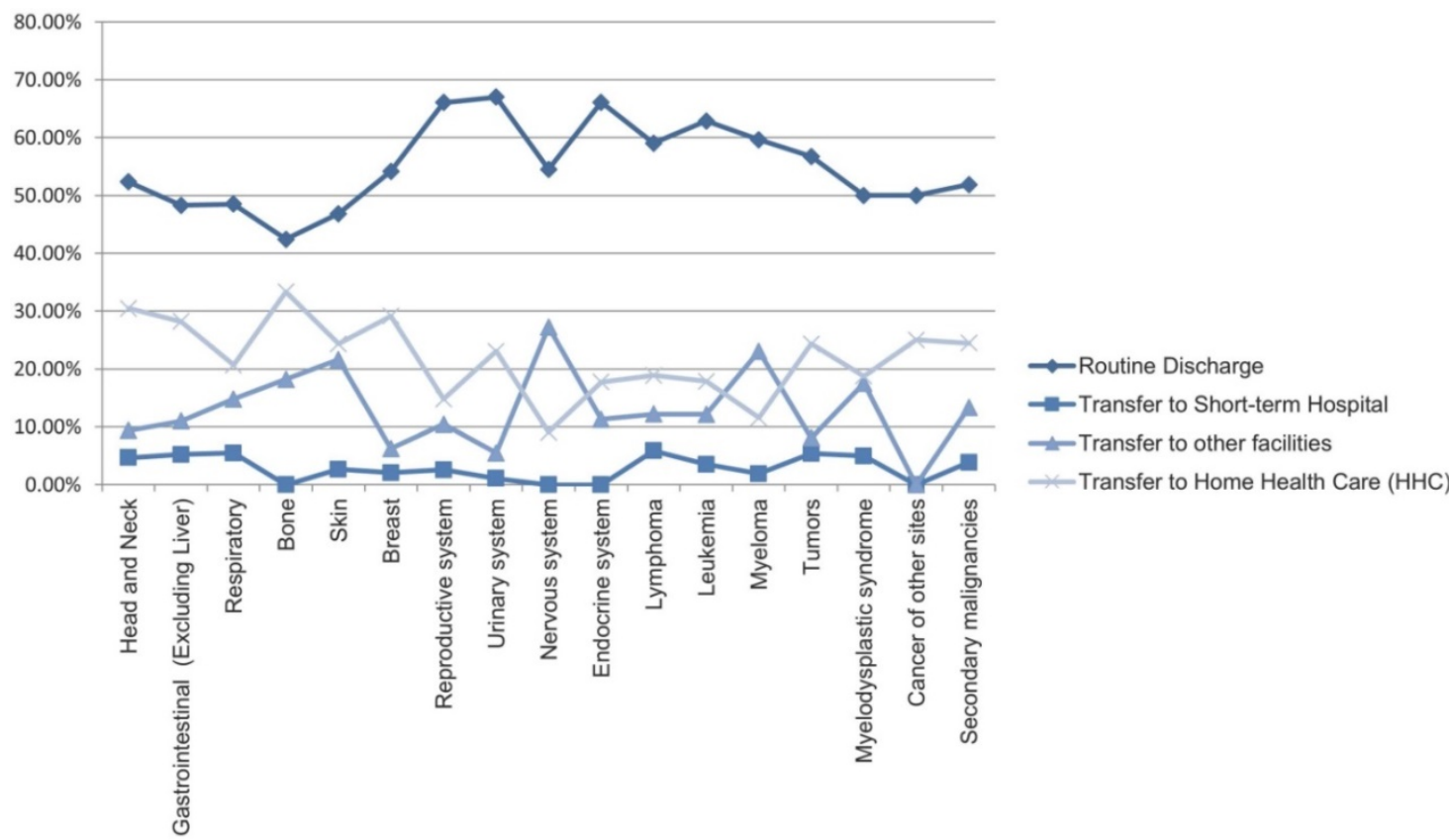

Figure 3: Bar Plots of Length of Stay (mean and standard deviation) of all Malignant Neoplasms in LTX Patients.

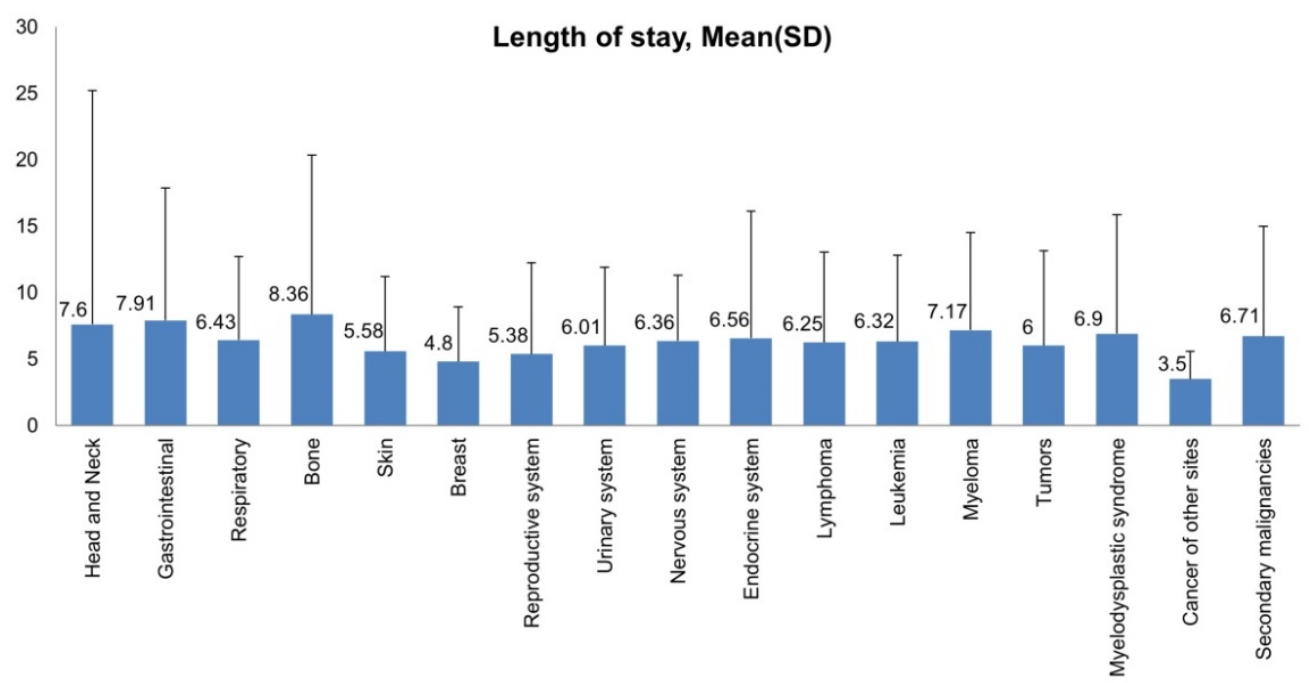

Figure 4: Bar Plots of Mean Total Charges of all Malignant Neoplasms in LTX Patients.

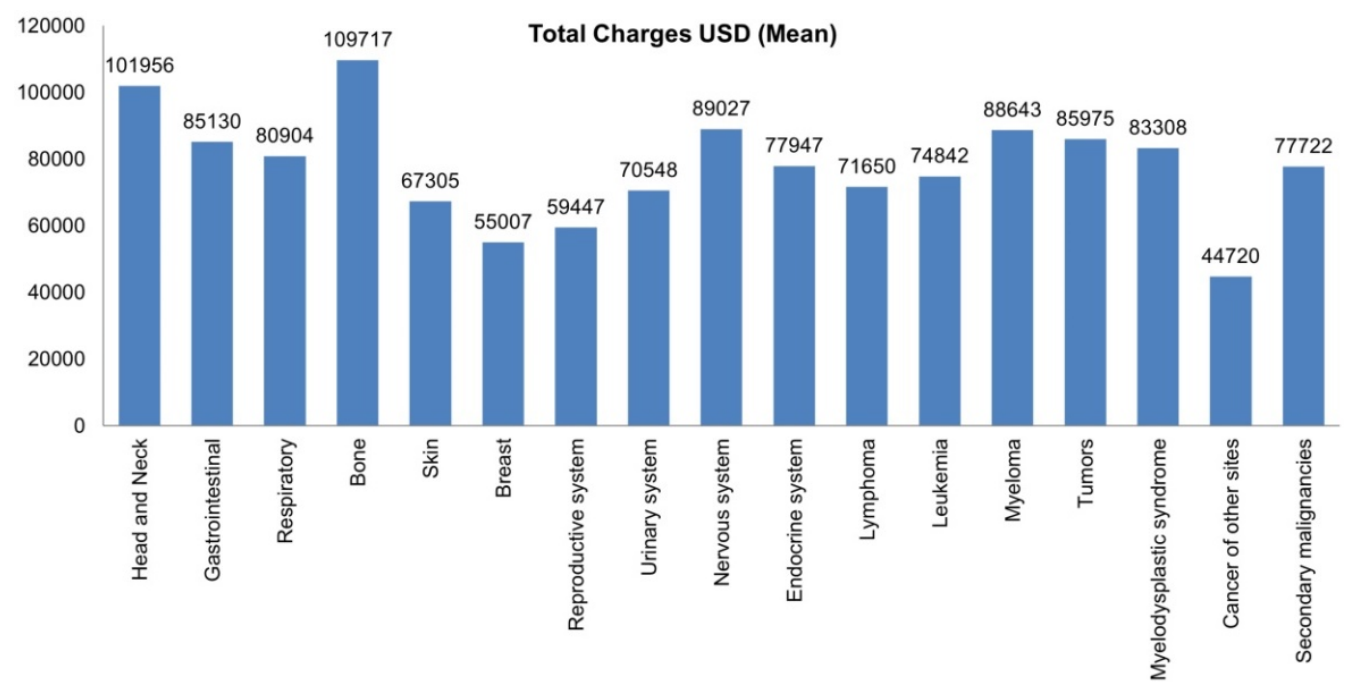

Figure 5: Bar Plots of in-hospital Mortality all Malignant Neoplasms in LTX Patients. 
In hospital mortality

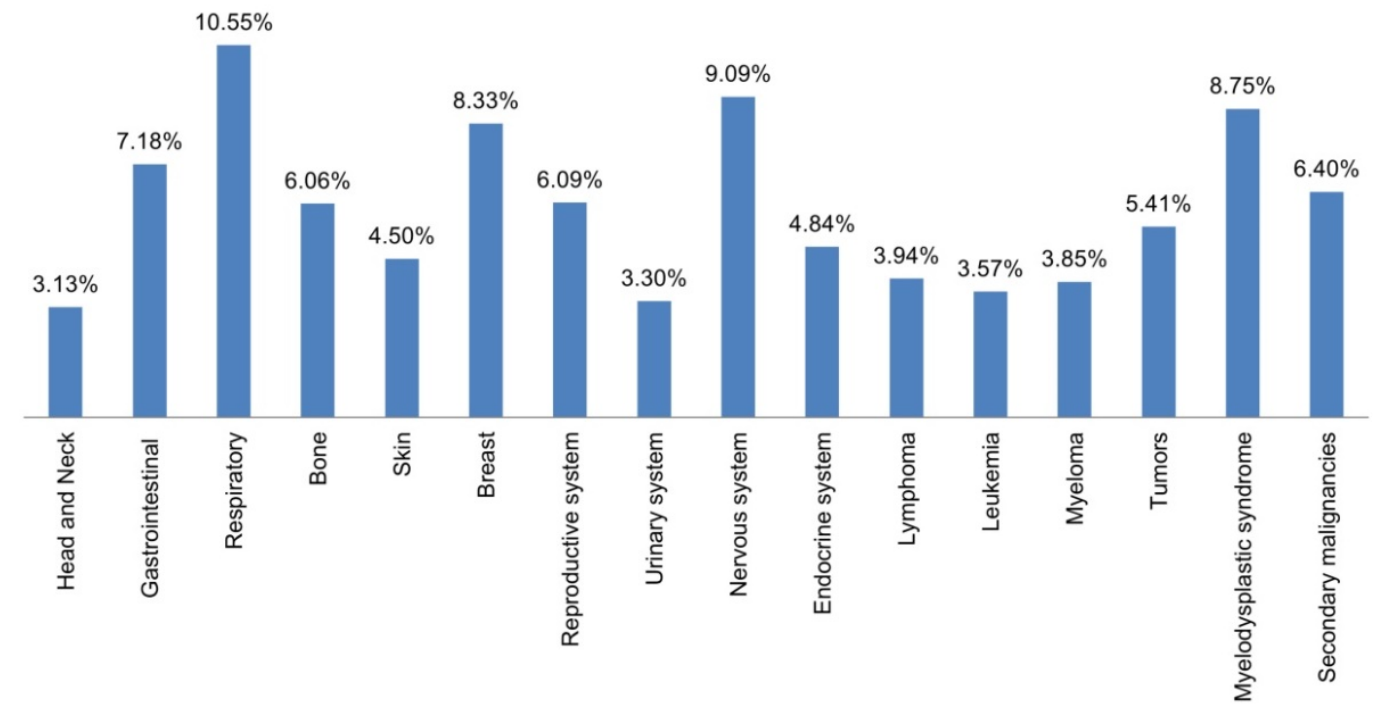

Figure 6: Line Graph of Discharge Rate (Proportions) of all Malignant Neoplasms in LTX Patients.

Table 4: Incidence of Malignant Neoplasms in LTX patients with Vs. Matched cohort of non-LTX patients.

\begin{tabular}{|c|c|c|c|}
\hline \multirow[t]{2}{*}{ Variables } & \multicolumn{2}{|c|}{$\begin{array}{l}\text { 1:1 matched comparison of LTX \& } \\
\text { non-LTX patients }\end{array}$} & \multirow[t]{2}{*}{$P$ value } \\
\hline & $\begin{array}{l}\text { LTX }=\text { No } \\
(n=26225) 50 \%\end{array}$ & $\begin{array}{l}\text { LTX }=\text { Yes } \\
(\mathrm{n}=26225) 50 \%\end{array}$ & \\
\hline Head and neck & $87(0.33 \%)$ & $128(0.49 \%)$ & 0.005 \\
\hline Cardiac & $3(0.01 \%)$ & $0(0.00 \%)$ & NS \\
\hline Gastrointestinal & $375(1.43 \%)$ & $209(0.80 \%)$ & $<.0001$ \\
\hline Respiratory & $338(1.29 \%)$ & $237(0.90 \%)$ & $<.0001$ \\
\hline Bone & $48(0.18 \%)$ & $33(0.13 \%)$ & 0.0953 \\
\hline Skin & $33(0.13 \%)$ & $111(0.42 \%)$ & $<.0001$ \\
\hline Breast & $95(0.36 \%)$ & $48(0.18 \%)$ & $<.0001$ \\
\hline Reproductive & $268(1.02 \%)$ & $115(0.44 \%)$ & $<.0001$ \\
\hline Urinary & $140(0.53 \%)$ & $91(0.35 \%)$ & 0.0012 \\
\hline Nervous system & $70(0.27 \%)$ & $11(0.04 \%)$ & $<.0001$ \\
\hline Endocrine & $98(0.37 \%)$ & $62(0.24 \%)$ & 0.0044 \\
\hline Lymphoma & $182(0.69 \%)$ & $254(0.97 \%)$ & 0.0005 \\
\hline Leukemia & $204(0.78 \%)$ & $140(0.53 \%)$ & 0.0005 \\
\hline Myeloma & $86(0.33 \%)$ & $52(0.20 \%)$ & 0.0038 \\
\hline Tumors & $24(0.09 \%)$ & $37(0.14 \%)$ & 0.0958 \\
\hline Myelodysplastic syndrome & $50(0.19 \%)$ & $80(0.30 \%)$ & 0.0084 \\
\hline Cancers of other sites & $6(0.02 \%)$ & $4(0.02 \%)$ & NS \\
\hline Secondary malignancies & $764(2.91 \%)$ & $719(2.74 \%)$ & 0.2359 \\
\hline
\end{tabular}

${ }_{1}^{1}$ Pearson Chi-Square 2-tailed Test for association of two Categorical Variables. NS: Not statistically significant.

\section{Discussion}

Previous studies demonstrated that liver transplant recipients were at risk of developing de novo malignancies $[2,3,4,5,6,7,8,13,14,23,24,26]$. Fewer studies reported a 3-7 times greater risk for malignancy among LTX recipients than the general population [13]. Based on the data from the literature, the incidence rate of malignancies after liver transplant ranged from $4 \%$ to $16 \%$. Our study found a total of 26225 hospital admissions with liver transplant status occurred in the three years from 2016 to 2018, of which 1909 admissions (7.28\%) had malignant neoplasms excluding hepatocellular carcinoma. The most common reported malignancy among admitted patients was lymphoma, followed by respiratory cancers and gastrointestinal cancers. Based on the literature, the hazard of developing malignancy is one out of every six patients within 20 years of liver transplantation [14]. Previous studies discussed multiple risk factors related to the occurrence of malignancies in post-transplant patients. They included the following: recipient age, excess immunosuppression, alcohol liver disease, smoking, hepatitis B, hepatitis C, HIV, Kaposi sarcoma, and EBV seropositivity of donor and recipient $[13,28]$. In our study, smoking, Alcoholic cirrhosis, Hepatitis B, and Hepatitis C, were statistically significant risk factors of post-liver transplantation malignancies. Although, more opioid use was seen among the malignant cohort. Among virus-related malignancies, non-Hodgkin lymphomas were the most reported neoplasms in liver transplant patients [3]. In our study, LTX patients with long-term immunosuppressive agents were at a higher risk of malignant neoplasm $(0.84 \%$ among cases vs. $0.75 \%$ among control). However, they were not statistically significant, possibly due to fewer patients and limitations of the NIS dataset. In addition, we could not compare necessary information like the blood concentration, dose, and the duration of the immunosuppressants. Immunosuppressive therapy in organ transplant recipients predisposes them to cancer by downplaying the immune response against the malignant antigens. Immunosuppressant has dose-dependent effects; the higher the drug dose, the higher the risk of de novo malignancy or recurrence of hepatocellular carcinoma [15, 22]. Few in-vitro studies [16] showed that anti-rejection drugs could have 
pro-oncogenic activity by overexpressing the transforming growth factor-beta (TGF-ß), causing impairment of signaling pathways and dysregulation of the immune system, thus, promoting the development and progression of aggressive malignancy [27]. Hence, similarly to the dose-effect, the longer the duration of immunosuppressive agents, the higher the risk for malignancies [17]. Viral infections constitute another critical risk factor for neoplasm development. Viruses modulate and disrupt the normal cell-cycle functions of the host cells; their encoded viral genes sabotage the immune system, thus, increase the risk of tumorigenesis [18]. In the United States, the incidence of malignancy transmission from deceased donors is < $1 \%$ [19].

Our study showed that the risk of developing malignant neoplasms in LTX patients increases with age. Also, malignant neoplasms were more common in males than females (29\% lesser odds- aOR, 0.712; $95 \%$ CI, $0.643-0.788 ; \mathrm{p}<.001)$. Black and Hispanic patients were at lower risk than Whites. Our study also showed that smoking is a significant risk factor associated with higher odds of malignant neoplasms in LTX patients. 12\% greater odds to develop cancers with smoking (aOR, 1.121; 95\% CI, 1.013 - 1.240; $\mathrm{p}<$ .05 . LTX patients had $36 \%$ greater odds of opioids use (aOR, 1.362; 95\% CI, 1.056 - 1.756; $\mathrm{p}<.01$ ).

\section{Post-transplant Lymphoproliferative Disease}

Our study revealed that the most common malignancies present among hospitalized LTX patients are lymphomas, followed by respiratory cancers. Gastrointestinal cancers, excluding hepatocellular carcinoma, marked the third top malignancy. In our population dataset, lymphomas accounted for $0.97 \%$ of admissions in post-liver transplant patients, with the highest incidence among Hispanics (20.27\%). lymphomas or lymphoproliferative disorders in the post-transplant phase could correlate with inadequate immune response toward the EBV virus [15]. Other responsible viruses are hepatitis $B$ and hepatitis $C$ for liver cancer and HPV in squamous cell carcinoma [28]. One of the protective factors for lymphoma and leukemia is to use alternative immunosuppressive agents like mycophenolate mofetil [20].

\section{Respiratory Cancers}

Respiratory malignant neoplasms are the second leading in our LTX patient's cohort. Often lung cancer is seen in lung transplant patients or heart transplant recipients [21]. The incidence of lung or bronchus cancer in organ transplant recipients is higher among smokers- as expected. Some studies have suggested a potential association between increased risk for respiratory cancers and the use of immunosuppression with anti-lymphocyte globulins for induction therapy and rejection prevention after transplantation [22]. Based on our results, $0.90 \%$ of LTX patients developed respiratory/lung cancer, which was the most common neoplasm in Whites, accounting for $15.78 \%$.

\section{Gastrointestinal Malignancies}

Gastrointestinal cancers, excluding hepatocellular carcinomas, ranked third as the most common malignancies in our study population. Out of all the GI malignancies, colorectal malignancies in LTX patients were the most studied. Previous studies showed that the incidence rate of colorectal cancer is 0.4 to $0.54 \%$ in the United States [23]. Our study showed that $0.8 \%$ of LTX patients' admissions were related to GI malignancies (excluding HCC). It is the second most common cancer among Hispanics $(13.51 \%)$. The literature revealed that patients with primary sclerosing cholangitis (PSC) associated with inflammatory bowel disease (IBD) are at high risk for developing GI malignancies [26]. The exact pathogenesis for increasing the risk of colorectal cancer with PSC is not known. One hypothesis is that inflammation can expedite the process. Patients with PSC and IBD require more aggressive immunosuppression, thus inadvertently risking any de novo malignancy [24].

\section{Other Malignancies}

Other cancers observed in LTX patients included head and neck, genitourinary, nervous system, bone, skin, leukemia, myeloma, breast, and endocrine cancers. Data on admissions with cardiac cancers were missing. Head, neck, and bone cancers had lesser admission rates $(0.49 \%$ and $0.13 \%)$, but their inpatient hospital charges were higher, and their length of stay was more extended than more prevalent cancers like lymphomas.

\section{Comparative analysis of Incidence of Malignant Neoplasms in LTX patients and matched Cohort of Non-LTX patients}

Our study revealed that LTX patients are at increased risk of head and neck cancers, skin cancers, lymphoma, tumors, and Myelodysplastic syndrome compared to our non-LTX hospitalized patient cohort. The highest on the list is skin cancer with 3.2 times higher odds, which aligns with the previous studies' results [25], followed by myelodysplastic syndrome with 1.58 times higher odds. Head and Neck cancers and lymphomas are third and fourth on the list.

\section{Hospital Burden and Mortality}

This study highlighted that most admissions of LTX patients with malignancy were elective and were on weekdays. Patients preferred sizeable urban teaching hospitals over small urban non-teaching 
hospitals. LTX patients with malignant neoplasms had a longer length of stay with a mean of 6.45 days as compared to 5.56 days of LTX patients with no malignancies. This longer length of stay can be attributed to more comorbidities and complications in this patient population. Our results showed that bone cancers had the most extended length of stay, possibly because of widespread metastasis and malignancy complications. In-hospital mortality was 3.3 times higher in LTX patients with malignant neoplasms than in those without malignancy. Respiratory cancers accounted for the highest inpatient mortality. Inpatient hospital cost of care was higher in LTX patients with malignant neoplasms secondary to more complicated hospital courses and expensive chemotherapy treatment regimens- thus increasing the health care burden. Out of all malignancies, bone cancers recorded the highest utilization of hospital charges. This finding is in line with our previously mentioned observation of bone cancer patients' most extended length of stay.

\section{Limitations and strengths}

We used the National Inpatient Sample database with data collected between January 2016 and December 2018. With more than 7 million records per year, the NIS administrative database could be prone to selection bias and coding errors. Our study encountered multiple limitations; for instance, liver donor information was lacking from the database, and one of the significant risk factors of malignancy in post LTX patients includes EBV seropositivity in the donor.Another limitation was the missing data regarding the length of time (the interval duration) between transplantation and the incidence of diagnosis of malignancy. In addition, the duration and dosage of immunosuppressant drugs were not available. Moreover, since this database housed inpatients data only, we could not evaluate any outpatient follow-up and previous cancer screening. Therefore, the calculated mortality from malignant neoplasms reflects only that for the inpatient sample and does not account for outpatient mortality rates; therefore, it could be that our data present an underestimation of malignancy-associated mortality rates post liver transplant.

This study remains unique because it highlights the impact of malignancy post-liver transplant using a large and diverse cohort of patients with national data from all United States regions compared to previous studies of specific geographical areas or centers. In addition, we compared the LTX patients with a non-LTX matched cohort and compared the incidence of various cancers. To the best of our knowledge, it is the first study from the National Inpatient Sample database to analyze hospital utilization and the discharge status of all liver transplant patients. Despite the inherent pitfalls of using an administrative database to study clinical outcomes, still, the NIS database houses a plethora of national data that provide a rough estimation of the cumulative prevalence of malignancy post-liver transplant at the national level; thus, its utilization can be harnessed to generate new hypotheses for prospective controlled experimental studies.

\section{Conclusion}

The number of post-liver transplantation complications is on the rise. Therefore, our study aimed to observe the incidence of malignancies in hospitalized liver transplant patients. Among these hospitalized LTX patients, lymphoproliferative diseases followed by respiratory cancers were the most reported malignancies. Moreover, our research found that LTX hospitalized patients are at increased risk of head and neck cancers, skin cancers, lymphoma, tumors, and Myelodysplastic syndrome than non-LTX patients.

This study highlights a clear trend in the patient sociodemographic and discharges characteristics. Advanced age, being White, and being male were significantly associated with a higher likelihood of malignant neoplasms in liver transplant patients. Patients aged 70 and up were 2.7 times more likely to develop malignancy than young patients; females had $29 \%$ lesser odds of malignancy than males.

In our research, factors like smoking, Alcoholic cirrhosis, Hepatitis B, Hepatitis C were statistically significant risk factors of post-liver transplantation malignancies. Moreover, our study also found opioids linked with a higher rate of post-liver transplantation malignancies.

Respiratory cancer had the highest risk of mortality among all cancers, accounted for $10.5 \%$ of all reported mortalities. Cancers of the nervous system, myelodysplastic syndrome, and breast cancer admission rates were lower, but their mortality was higher than other cancers with $9.1 \%, 8.75 \%$, and $8.3 \%$. Although head, neck, and bone cancers were uncommon in LTX patients, they had a longer length of stay and hospital charges than more frequent cancers like lymphomas and respiratory. Most of the patients had routine discharge, followed by transfer to home health care, other facilities (Includes Skilled Nursing Facility, Intermediate Care Facility), or short-term hospitals.

To conclude, regular cancer screening in liver transplant patients is crucial as early detection helps in enhancing treatment responsiveness. Therefore, clinicians should proactively seek to diagnose these 
aggressive cancers post-liver transplantation as early as possible to reduce morbidity and mortality, hospital burden, and improve patients' outcomes and quality of life.

\section{Author Contributions}

The authors confirm contribution to the paper as follows:

Study conception and design: Author, Maryam Bilal Haider (corresponding author). Data collection and statistical analysis: Author, Syed M Haider. Interpretation of results: Author, Rana Ismail. Draft manuscript preparation: Author, Maryam Bilal Haider, and Anusha Bapatla. Agreement to be accountable for all aspects of the work in ensuring that questions related to the accuracy or integrity of any part of the work are appropriately investigated and resolved: Author, Rana Ismail, Ahmed J Chaudhary, and Sana Iqbal.

All authors reviewed the results and approved the final version of the manuscript.

\section{Competing Interests}

The authors have declared that no competing interest exists.

\section{References}

1. Cosme Manzarbeitia MD. Liver Transplantation [Internet]. Practice Essentials, History of the Procedure, Epidemiology. Medscape; 2019 [cited 2021Feb22]. Available from: https://emedicine.medscape.com/article/431783-overview

2. Dumortier J, Maucort-Boulch D, Poinsot D, Thimonier E, Chambon-Augoyard $\mathrm{C}$, Ducroux E, et al. Immunosuppressive regimen and risk for de novo malignancies after liver transplantation for alcoholic liver disease. Clin Res Hepatol Gastroenterol. 2018;42(5):427-35.

3. Taborelli M, Piselli P, Ettorre GM, Baccarani U, Burra P, Lauro A, et al. Survival after the diagnosis of de novo malignancy in liver transplant recipients: Cancer survival following liver transplant. Int J Cancer. 2019;144(2):232-9.

4. Martin HL, Chen JWC, Koczwara B. Cancer in liver transplant recipients: management and outcomes: Cancer in liver transplant recipients. Asia Pac J Clin Oncol. 2013;9(3):257-64

5. Schrem H, Kurok M, Kaltenborn A, Vogel A, Walter U, Zachau L, et al. Incidence and long-term risk of de novo malignancies after liver transplantation with implications for prevention and detection: Cancer After LT: Incidence, Prevention, Detection. Liver Transpl. 2013;19(11):1252-61.

6. Fung JJ, Jain A, Kwak EJ, Kusne S, Dvorchik I, Eghtesad B. De novo malignancies after liver transplantation: a major cause of late death. Liver Transpl. 2001;7(11 Suppl 1):S109-18

7. Kang EA, Koh S-J, Kim JW, Lee KL, Im JP, Kim JS, et al. Prevalence of advanced colorectal neoplasm is higher in liver transplant recipients. Turk J Gastroenterol. 2018;29(3):316-24

8. Manzia TM, Angelico R, Gazia C, Lenci I, Milana M, Ademoyero OT, et al. De novo malignancies after liver transplantation: The effect of immunosuppression-personal data and review of literature. World J Gastroenterol. 2019;25(35):5356-75.

9. Zimmerman MA, Ghobrial RM, Tong MJ, Hiatt JR, Cameron AM, Hong J, et al. Recurrence of hepatocellular carcinoma following liver transplantation: a review of preoperative and postoperative prognostic indicators: A review of preoperative and postoperative prognostic indicators. Arch Surg. 2008;143(2):182-8; discussion 188

10. HCUP National Inpatient Sample (NIS). Healthcare Cost and Utilization Project (HCUP). 2012. Agency for Healthcare Research and Quality, Rockville, MD. www.hcup-us.ahrq.gov/nisoverview.jsp Accessed February 22, 2021.

11. HCUP Clinical Classifications Software (CCS) for ICD-10-CM. Healthcare Cost and Utilization Project (HCUP). November 2020. Agency for Healthcare Research and Quality, Rockville,MD. www.hcup-us.ahrq.gov/toolssoftware/ ccs/ccs.jsp. Accessed February 22, 2021.

12. DUA Training - Accessible Version. Healthcare Cost and Utilization Project (HCUP). April 2020. Agency for Healthcare Research and Quality, Rockville,
MD. www.hcup-us.ahrq.gov/DUA/dua_508/DUA508version.jsp Accessed February 22, 2021.

13. Wahab MA, Abdel-Khalek EE, Elshoubary M, Yassen AM, Salah T, Sultan $\mathrm{AM}$, et al. Predictive factors of $D E$ Novo malignancies after living-donor liver transplantation: A single-center experience. Transplant Proc. 2021;53(2):63644 .

14. Aberg F, Pukkala E, Höckerstedt K, Sankila R, Isoniemi H. Risk of malignant neoplasms after liver transplantation: a population-based study. Liver Transpl. 2008;14(10):1428-36.

15. Taborelli M, Piselli P, Ettorre GM, Lauro A, Galatioto L, Baccarani U, et al. Risk of virus and non-virus related malignancies following immunosuppression in a cohort of liver transplant recipients. Italy, 1985-2014. Int J Cancer. 2018;143(7):1588-94.

16. Maluccio M, Sharma V, Lagman M, Vyas S, Yang H, Li B, et al. Tacrolimus enhances transforming growth factor-beta1 expression and promotes tumor progression. Transplantation. 2003;76(3):597-602.

17. Huo Z, Li C, Xu X, Ge F, Wang R, Wen Y, et al. Cancer risks in solid organ transplant recipients: Results from a comprehensive analysis of 72 cohort studies. Oncoimmunology. 2020;9(1):1848068.

18. Guba M, Graeb C, Jauch K-W, Geissler EK. Pro- and anti-cancer effects of immunosuppressive agents used in organ transplantation. Transplantation. 2004;77(12):1777-82

19. Nalesnik M, Ison. Organ transplantation from deceased donors with cancer: is it safe? Open Access Surg. 2011;4:11.

20. Ohsugi Y, Suzuki S, Takagaki Y. Antitumor and immunosuppressive effects of mycophenolic acid derivatives. Cancer Res. 1976;36(8):2923-7.

21. Tsai H-I, Lee C-W, Kuo C-F, See L-C, Liu F-C, Chiou M-J, et al. De novo malignancy in organ transplant recipients in Taiwan: a nationwide cohort population study. Oncotarget. 2017;8(22):36685-95.

22. Piselli P, Verdirosi D, Cimaglia C, Busnach G, Fratino L, Ettorre GM, et al. Epidemiology of de novo malignancies after solid-organ transplantation: immunosuppression, infection and other risk factors. Best Pract Res Clin Obstet Gynaecol. 2014;28(8):1251-65.

23. Silva MA, Jambulingam PS, Mirza DF. Colorectal cancer after orthotopic liver transplantation. Crit Rev Oncol Hematol. 2005;56(1):147-53.

24. Mouchli MA, Singh S, Loftus EV, Boardman L, Talwalkar J, Rosen CB, et al. Risk factors and outcomes of DE Novo cancers (excluding nonmelanoma skin cancer) after liver transplantation for primary sclerosing cholangitis. Transplantation. 2017;101(8):1859-66.

25. Euvrard S, Kanitakis J, Claudy A. Skin cancers after organ transplantation. N Engl J Med. 2003;348(17):1681-91.

26. Fabia R, Levy MF, Testa G, Obiekwe S, Goldstein RM, Husberg BS, et al. Colon carcinoma in patients undergoing liver transplantation. Am J Surg. 1998;176(3):265-9.

27. Kubiczkova L, Sedlarikova L, Hajek R, Sevcikova S. TGF- $\beta$ - an excellent servant but a bad master. J Transl Med. 2012;10(1):183

28. Chandok N, Watt KD. Burden of de novo malignancy in the liver transplant recipient. Liver Transpl. 2012;18(11):1277-1289. 